\section{KIM-1 driving chronic kidney disease}

\section{By Benjamin Boettner, Assistant Editor}

Kidney injury molecule 1, a protein that helps overcome acute kidney injury, now has been shown by Harvard Medical School researchers to trigger the onset of chronic kidney disease. ${ }^{1}$ Companies modulating the target thus need to scrutinize the therapeutic window of their molecules.

The body typically repairs nonfibrotic kidney damage inflicted by acute toxic or ischemic insults without long-term sequelae. However, some patients who recover from an acute kidney injury are left with an increased risk for developing chronic kidney disease (CKD), which manifests itself by fibrosis, impaired kidney parenchyma and cardiovascular malfunction. ${ }^{2}$

Kidney injury molecule 1 (KIM-1) is a cell surface receptor that is expressed on kidney epithelial cells and by distinct $\mathrm{T}$ cell populations and hepatocytes. In acutely injured kidneys, KIM-1 is thought to confer a phagocytic phenotype on kidney epithelial cells that contributes to recovery by removing debris from cells that died during the injury. ${ }^{3}$

In patients with CKD, on the other hand, elevated KIM-1 expression has been shown to occur close to inflammatory cells and fibrotic tissue. ${ }^{4}$ However, whether KIM-1 actively promotes kidney fibrosis has not been resolved. The fibrotic reaction at the heart of CKD is triggered by inflammation-induced proliferation and recruitment of myofibroblasts that deposit extracellular matrix proteins. Those proteins, in turn, obstruct the kidney parenchyma.

To shed some light on the mechanistic role of KIM-1 in CKD, a team led by Benjamin Humphreys and Joseph Bonventre first used an established model of fibrosis to determine the dynamics of KIM-1 expression after acute injury in the absence of fibrosis. They showed that KIM-1 levels rapidly spike in surviving proximal epithelial cells, subside during recovery but, importantly, remain above normal thereafter.

Humphreys is an assistant professor in the department of medicine at Harvard Medical School and an associate physician of medicine at Brigham and Women's Hospital. Bonventre is a professor at Harvard Medical School and chief of the renal unit and director of the bioengineering division at Brigham and Women's Hospital.

Next, the researchers created a mouse model in which Kim-1 was overexpressed in kidney epithelial cells. The animals developed tubulointerstitial inflammation and fibrosis six weeks after birth and died of progressive renal failure about five weeks later.

"The genetic model does not require injury to trigger the pathogenic changes leading to CKD," noted Oxana Ibraghimov-Beskrovnaya, VP of cell biology and distinguished scientific fellow at Sanofi's Genzyme Corp. unit. "The model should be uniquely useful for studying the role of a maladaptive KIM-1 response in kidney fibrosis as well as for testing novel therapeutic modalities."

Similar to human CKD, fibrotic disease in the mice was accompanied by cardiac remodeling and hypertension.

Mechanistically, overexpression of Kim-1 in epithelial cells induced proinflammatory cytokines, which triggered the recruitment of macrophages into the interstitial lumen and the initiation of the fibrotic process.

In a model of fibrosis, mice producing a partial loss-of-function version of Kim-1 did not develop the condition.

Humphreys told SciBX that the data show that "persistent KIM-1 expression clearly is harmful."

He thinks that the findings may help explain why humans, in particular the elderly, often develop CKD following an episode of acute kidney injury.

"We hypothesize that KIM-1 induction after an episode of acute kidney injury is not downregulated in some patients, and this causes a simmering maladaptive repair response characterized by a proinflammatory environment that ultimately causes parenchymal loss, fibrosis and CKD," he said.

"The general notion of KIM-1 as a protective protein in the kidney has to be revised now, and in fact the study shows that KIM-1 exerts a bimodal function in a very organized fashion during disease progression in mice. It will have to be seen now whether this is also the case in humans," said Raghu Kalluri, chair of the Department of Cancer Biology at The University of Texas MD Anderson Cancer Center.

The findings were published in Nature.

\section{All aKIMbo}

Humphreys' team plans to use conditional models to overexpress Kim-1 exclusively in adult mice. He said that this should more closely emulate what happens in humans after an episode of acute injury and should help distinguish the beneficial effects of KIM-1 in the acute setting and its negative effects in the chronic arena.

Humphreys thinks that any attempts to target KIM-1 may be more complicated than simply inhibiting all of its function.

"In acute kidney injury, KIM-1 function is good, in CKD it becomes bad-that is what makes drug development challenging," agreed Kalluri.

In addition, KIM-1 is expressed in other organs like the lung or liver. Thus, said Kalluri, "systemic inhibition of KIM-1 could cause unanticipated effects at these sites." 


\section{ANALYSIS}

\section{TARGETS \& MECHANISMS}

To validate KIM-1 as a viable target in CKD, Paul Rennert wanted to see "a study to show that kidney injury leading to fibrosis is reduced or ablated in Kim-1 knockout mice. A similar study could be done using specific anti-mouse Kim-1 mAbs to block Kim-1 activity in normal mice subjected to acute kidney injury."

Such evidence would unequivocally show that KIM-1 is detrimental to regeneration in CKD. Rennert is CSO of $\mathbf{X}$ - Rx Discovery Inc. $\mathrm{He}$ previously was a principal investigator at Biogen Idec Inc., where he studied the role of KIM-1 in immunity and infections.

Indeed, the expression of KIM-1 in immune cells could further complicate discovery efforts in kidney disease. "Since KIM-1 expressed by cells of the immune system can bind to KIM-1 expressed by the transgene, it becomes difficult to fully understand the role of the immune system in the presented mouse model," said Rennert.

He suggested that strain-matched Kim-1 knockout immune cells could be used in an adoptive transfer model to distinguish the immune system and the stromal effects of Kim-1 expression in renal injury and fibrosis models.

Such studies "could be complemented by mAb studies in wild-type rodents that would inhibit KIM-1 more broadly across different cell types," he said.

The Harvard team holds issued patents on KIM-1 for diagnostics and therapeutics and has filed additional patent applications based on studies in mouse and zebrafish models of diabetic nephropathy kidney disease.

The findings are available for licensing, and KIM-1 patents have been licensed by Johnson \& Johnson, Genzyme, Biogen Idec and undisclosed companies.

Boettner, B. SciBX 6(39); doi:10.1038/scibx.2013.1085

Published online Oct. 10, 2013

\section{REFERENCES}

1. Humphreys, B.D. et al. J. Clin. Invest.; published online Aug. 27, 2013; doi:10.1172/JCl45361

Contact: Benjamin D. Humphreys, Brigham and Women's Hospital, Boston, Mass. e-mail: bhumphreys@partners.org

2. Leung, K.C.W. et al. Nat. Rev. Nephrol. 9, 77-85 (2013)

3. Ichimura, T. et al. J. Clin. Invest. 118, 1657-1668 (2008)

4. van Timmeren, M.M. et al. J. Pathol. 212, 209-217 (2007)

COMPANIES AND INSTITUTIONS MENTIONED

Biogen Idec Inc. (NASDAQ:BIIB), Weston, Mass.

Brigham and Women's Hospital, Boston, Mass.

Harvard Medical School, Boston, Mass.

Genzyme Corp., Cambridge, Mass.

Johnson \& Johnson (NYSE:JNJ), New Brunswick, N.J.

Sanofi (Euronext:SAN; NYSE:SNY), Paris, France

The University of Texas MD Anderson Cancer Center, Houston, Texas

X-Rx Discovery Inc., Waltham, Mass. 